years ${ }^{2,4}$. Earlier writers proposed structures involving rings, such as $\mathrm{I}^{3,4}$ or $\mathrm{II}^{5}$, or quinonoid forms, such as III $^{6}$. Hodgson and Marsden ${ }^{2}$ conclude, however, that both the $o$ - and the $p$ - series are best regarded as resonance hybrids of the type

$$
\overline{\mathrm{O}}-\mathrm{C}_{6} \mathrm{H}_{4}-\stackrel{+}{\mathrm{N}} \equiv \mathrm{N} \longleftrightarrow \mathrm{O}=\mathrm{C}_{6} \mathrm{H}_{4}=\stackrel{+}{\mathrm{N}}=\overline{\mathrm{N}} \text {. }
$$

If such a view were correct, it is obvious from IV and $V$ qualitatively that, while the polarity of a $p$-diazo-oxide could be small, that of its o-isomer should be large, and-in proportion as the ionized form predominates-approach that of a true 'zwitterionic' molecule? . Thus the dipole moment of IV is unlikely to be less than c. $10 D$, while $\mathrm{V}$, on reasonable analogies, might be so low as $4 D$.

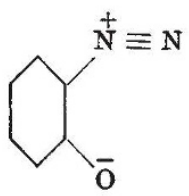

(IV)

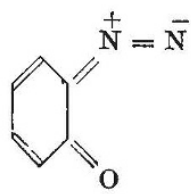

(V)<smiles>O=C1CCC(Br)CC1=[V]</smiles>

(VI)
Although we have not been able to prepare the unsubstituted parents IV or V, dipole moment determinations on representative derived members of both the $o$ - and the $p$-series are now nearing completion. Results are proving close to those expected for $V$ (that is, also III) ; for example, $\mu$ (found) for VI is $2.9 D$ (calculated, about $3 D$ ). Ultra-violet spectroscopic evidence is in the same direction.

Very little contribution from fully dipolar forms seems, therefore, to be occurring. We make this preliminary communication because we note that dipolar structures for the o-diazo-oxides have been adopted recently by Vaughan and Phillips ${ }^{8}$ to explain stability measurements.

Chemistry Department, University of Sydney. May 18

1 Calderbank, Le Fèvre and Nort hcott, J. Soc. Chem. Ind., 158 (1948). Hodgson and Marsden, J. Soc. Dyers. Col., 59, 271 (1943).

Bamberger, Ber., 28, 837 (1895).

- Morgan and Porter, J. Chem. Soc., 107645 (1915).

s.lemenc, Ber., 47, 1407 (1914).

- Wolff, Annalen, 312, 119 (1900).

'Wyman, Chem. Rev., 19, 213 (1936).

${ }^{8}$ J. Chem. Soc., 1566 (1947).

\section{Preparation of Nitrogen-free Sulphuric Acid}

OF the various methods suggested for the preparation of sulphuric acid suitable for use in nitrate estimations, the method of Atkins ${ }^{1}$ is perhaps the most suitable. There is, however, one disadvantage in this method, namely, the precipitation of sulphur if a slight excess of hydrogen sulphide is added. This not only takes a long time to settle, but also interferes with the estimation, often giving lower values due to the presence of traces of sulphur dioxide.

Various alternative methods were tried in this laboratory for the preparation of a suitable sample of sulphuric acid for the estimation of nitrates in seawater by the reduced strychnine method of Denigès. A good procedure seems to be to treat about $250 \mathrm{ml}$. of concentrated sulphuric acid (ordinary quality) with 6-10 drops of 40 per cent formalin solution in a $500 \mathrm{ml}$. Kjeldahl flask, and to boil the mixture until it is decolorized. It is possible to prepare about a litre of good acid in the course of a couple of hours by using a series of flasks. The acid so prepared gives an almost colourless reagent with reduced strychnine.

This method is, therefore, suggested as an alternative where difficulty is experienced in the preparation of acid according to Atkins' method.

\section{B. V. RAMACHANDRAN}

Central Marine Fisheries Research Station, Triplicane P.O., Madras.

April 24.

1 Atkins, W. R. G. Nature, 129, 98 (1982).

\section{Decay and Capture of Slow Mesons in Dielectrics}

Conversi, Pancini and Piccioni ${ }^{1}$, and others ${ }^{2}$, observed decay electrons from positive mesons coming to rest in solids of either light or heavy elements. For negative mesons, however, decay electrons were found in light elements only ; for heavy elements it is generally assumed that negative mesons are captured by atomic nuclei before they can decay. The absence of capture of positive mesons can be understood as being due to the influence of Coulomb repulsion ${ }^{3}$; its absence for negative mesons in light nuclei has led Fermi, Teller and Weisskopf ${ }^{4}$ to the conclusion that the interaction between nuclei and mesons is much weaker than was assumed hitherto. These authors show that it takes less than $10^{-12}$ sec. for a meson to reach an energy of about $1,000 \mathrm{eV}$., where its velocity is about equal to that of electrons in outer orbits, that is, $10^{8} \mathrm{~cm} . / \mathrm{sec}$. They then consider that the meson quickly loses its remaining energy, reaching the $K$-orbit of an atomic nucleus in about $10^{-12}$ sec. It should then be captured by the nucleus in about $10^{-18} \mathrm{sec}$. if current ideas of interaction between mesons and nuclei are correct. In doing so they have, however, considerably overestimated the energy transfer from slow mesons to electrons ${ }^{5}$. In the present communication reasons are given for believing that in dielectrics the time required by a meson to reach a $K$-orbit is longer than estimated by Fermi et al., but that it would be very difficult to decide from theoretical considerations whether this time may be of the same order as the decay time $\left(\sim 2 \times 10^{-6}\right.$ sec. $)$.

Mesons with a velocity less than about $10^{8} \mathrm{~cm} . / \mathrm{sec}$. (kinetic energy $<1,000 \mathrm{eV}$.) transfer energy to electrons (the velocity of which is greater than $10^{8} \mathrm{~cm}$. /sec.) at a very low rate (at least in non-metals) because the electrons can follow nearly adiabatically. (This would not hold for free electrons. Even metal electrons are not entirely free, and it seems, indeed, that their deviation from free electrons largely determines inelastic scattering. For, in view of the screening of the meson charge, the collision is of very short duration; development of the effective meson field into a Fourier series will lead mainly to high. frequency terms. The electrons then behave as bound, as for their optical properties.) However, in this region, down to an energy of about $0 \cdot 1 \mathrm{eV}$., the rate $B$ of loss of energy to the lattice vibrations is considerable. In ionic crystals, in particular, with residual frequency $\omega_{i} / 2 \pi$, it has been shown ${ }^{6}$ that: 